PROCEEDINGS OF THE

AMERICAN MATHEMATICAL SOCIETY

Volume 132, Number 8, Pages 2389-2397

S 0002-9939(04)07382-4

Article electronically published on February 20, 2004

\title{
A COUNTEREXAMPLE TO A CONJECTURE OF S. E. MORRIS
}

\author{
J. F. FEINSTEIN
}

(Communicated by N. Tomczak-Jaegermann)

\begin{abstract}
We give a counterexample to a conjecture of S. E. Morris by showing that there is a compact plane set $X$ such that $R(X)$ has no nonzero, bounded point derivations but such that $R(X)$ is not weakly amenable. We also give an example of a separable uniform algebra $A$ such that every maximal ideal of $A$ has a bounded approximate identity but such that $A$ is not weakly amenable.
\end{abstract}

\section{INTRODUCTION}

The notion of weak amenability for commutative Banach algebras was originally introduced in [1. A commutative Banach algebra $A$ is said to be weakly amenable if there are no nonzero, continuous derivations from $A$ into any commutative Banach $A$-bimodule. However, as shown in [1, it is enough to check the dual module $A^{\prime}$ : $A$ is weakly amenable if and only if there are no nonzero, continuous derivations from $A$ into $A^{\prime}$.

The condition of weak amenability sits between the stronger condition of amenability (as defined by B. E. Johnson in [13) and the weaker condition that the commutative Banach algebra $A$ have no nonzero, bounded point derivations (point derivations may be regarded as derivations into 1-dimensional, commutative Banach $A$-bimodules). There are examples in [1] of commutative, semisimple Banach algebras showing that no two of these three conditions are equivalent. (See also, for example, Sections 2.8 and 4.1 of [4] for further discussion of this area and many illustrative examples.)

For uniform algebras, the differences between these three conditions are less clear. Sheinberg's theorem (4, Theorem 5.6.2]) tells us that a uniform algebra $A$ on a compact Hausdorff space $X$ is amenable if and only if $A=C(X)$ (the uniform algebra of all continuous, complex-valued functions on $X)$. In this case we say that the uniform algebra $A$ is trivial.

No nontrivial, weakly amenable uniform algebras are known. There are examples of nontrivial, separable uniform algebras that have no nonzero, bounded point derivations ([17]) and even examples that have no nonzero point derivations at all ([5]). In this paper we show that such uniform algebras need not be weakly amenable.

We will assume some familiarity with the standard relationships between bounded approximate identities, strong boundary points, peak points and point

Received by the editors May 12, 2003.

2000 Mathematics Subject Classification. Primary 46J10, 46H20.

(C)2004 American Mathematical Society 
derivations for uniform algebras (especially for $R(X)$ ). Details may be found in the standard texts on uniform algebras (for example [3], 10] or [16]).

Notation. We denote the closed unit disc in $\mathbb{C}$ by $\bar{\Delta}$. More generally, $\Delta(a, r)$, $\bar{\Delta}(a, r)$ denote respectively the open, closed, discs of radius $r$ centered on $a$. For a bounded, complex-valued function $f$ defined on a nonempty set $S$ we shall denote by $|f|_{S}$ the uniform norm of $f$ on $S$; that is,

$$
|f|_{S}=\sup \{|f(x)|: x \in S\} .
$$

For a compact plane set $X, R_{0}(X)$ denotes the set of restrictions to $X$ of rational functions with poles off $X$. Thus the standard uniform algebra $R(X)$ is the uniform closure of $R_{0}(X)$ in $C(X)$.

Suppose that $\mu$ is a measure on a compact plane set $X$ such that the bilinear functional defined on $R_{0}(X) \times R_{0}(X)$ by

$$
(f, g) \mapsto \int_{X} f^{\prime}(x) g(x) \mathrm{d} \mu(x)
$$

is bounded. Then, as noted in [6], we may extend by continuity to $R(X) \times R(X)$ and obtain a continuous derivation $D$ from $R(X)$ to $R(X)^{\prime}$ satisfying, for $f$ and $g$ in $R_{0}(X)$,

$$
D(f)(g)=\int_{X} f^{\prime}(x) g(x) \mathrm{d} \mu(x) .
$$

Moreover, all continuous derivations from $R(X)$ to $R(X)^{\prime}$ have this form. Such a derivation is the zero derivation if and only if the measure $\mu$ annihilates $R(X)$.

In [15, S. E. Morris investigated the extent to which continuous derivations from $R(X)$ may be expressed as some form of integral of bounded point derivations. Morris conjectured that whenever $R(X)$ had no nonzero, bounded point derivations then $R(X)$ must be weakly amenable. We shall give a counterexample to this conjecture by constructing a suitable compact plane set $X$ such that $R(X)$ has no nonzero, bounded point derivations, but such that $R(X)$ is not weakly amenable. (Our example will be a "Swiss cheese".) In fact, we shall prove the following theorem.

Theorem 1.1. For each $C>0$ there is a compact plane set $X$ obtained by deleting from the closed unit disc a countable union of open discs such that the unit circle $\mathbb{T}$ is a subset of $X, R(X)$ has no nonzero, bounded point derivations, but for all $f$, $g$ in $R_{0}(X)$,

$$
\left|\int_{\mathbb{T}} f^{\prime}(z) g(z) \mathrm{d} z\right| \leq C|f|_{X}|g|_{X} .
$$

Let $X$ be one of the compact plane sets given by Theorem 1.1. In view of the discussion above there is a nonzero, continuous derivation $D$ from $R(X)$ into $R(X)^{\prime}$ satisfying, for all $f$ and $g$ in $R_{0}(X)$,

$$
D(f)(g)=\int_{\mathbb{T}} f^{\prime}(z) g(z) \mathrm{d} z .
$$

Thus $R(X)$ is not weakly amenable. Note, however, that the map $f \mapsto f^{\prime} \mid \mathbb{T}$ does not give a continuous derivation from $R(X)$ into $L^{1}(\mathbb{T})$, as the polynomial functions $f(z)=z^{n}$ show. 
The problem (for compact plane sets $X$ ) of whether $R(X)$ can be weakly amenable without being $C(X)$ remains open. If you allow derivations into completemetrizable modules, then the answer is negative: as shown in $[6], R(X)$ is "weakly$(F)$-amenable" if and only if it is trivial.

\section{Construction of the Swiss cheeses}

To construct our Swiss cheeses we require several lemmas.

The first lemma is similar to some standard estimates using Cauchy's theorem but has an extra twist using Fubini's theorem.

Lemma 2.1. Let $D_{n}$ be a sequence of open discs in $\mathbb{C}$ (not necessarily pairwise disjoint) whose closures are contained in the open unit disc. Set $X=\bar{\Delta} \backslash \bigcup_{n=1}^{\infty} D_{n}$. Let $s_{n}$ be the distance from $D_{n}$ to $\mathbb{T}$, and let $r_{n}$ be the radius of $D_{n}$. Let $f$ and $g$ be in $R_{0}(X)$. Then

$$
\left|\int_{\mathbb{T}} f^{\prime}(z) g(z) \mathrm{d} z\right| \leq 4 \pi|f|_{X}|g|_{X} \sum_{n=1}^{\infty} \frac{r_{n}}{s_{n}^{2}} .
$$

Proof. Let $\varepsilon>0$. For $N \in \mathbb{N}$ we set

$$
X_{N}=\bar{\Delta}(0,(N+1) / N) \backslash \bigcup_{n=1}^{N} D_{n}
$$

Then we may choose $N$ large enough that $f$ and $g$ have no poles in $X_{N}$ and

$$
4 \pi \frac{N+1}{N}|f|_{X_{N}}|g|_{X_{N}} \leq(4 \pi+\varepsilon)|f|_{X}|g|_{X}
$$

Using the boundary of $X_{N}$, and ignoring any isolated points (these may exist since the deleted discs can overlap), we obtain as usual (see, for example, pp. 28-29 of [2]) a contour made up of circular arcs which has winding number 1 around all points of the interior of $X_{N}$ and 0 around all points of $\mathbb{C} \backslash X_{N}$. We may split this contour into $\gamma_{1}$ and $\gamma_{2}$, where $\gamma_{1}$ is the circle centered on the origin, with radius $(N+1) / N$, while $\gamma_{2}$ is the rest. Note that $\gamma_{2}$ then satisfies

$$
\int_{\gamma_{2}} \frac{|\mathrm{d} \zeta|}{\operatorname{dist}(\zeta, \mathbb{T})^{2}} \leq \sum_{n=1}^{N} \frac{2 \pi r_{n}}{s_{n}^{2}}
$$

By Cauchy's formula, we have

$$
f^{\prime}(z)=h_{1}(z)+h_{2}(z)
$$

where $($ for $j=1,2)$

$$
h_{j}(z)=\frac{1}{2 \pi i} \int_{\gamma_{j}} \frac{f(w)}{(w-z)^{2}} \mathrm{~d} w
$$

We now have

$$
\int_{\mathbb{T}} f^{\prime}(z) g(z) \mathrm{d} z=\int_{\mathbb{T}} h_{1}(z) g(z) \mathrm{d} z+\int_{\mathbb{T}} h_{2}(z) g(z) \mathrm{d} z .
$$

We estimate the moduli of these two integrals separately.

The estimate for the $h_{2}$ integral is fairly standard: by (2) we have, for $z \in \mathbb{T}$,

$$
\left|h_{2}(z)\right| \leq|f|_{X_{N}} \sum_{n=1}^{N} \frac{r_{n}}{s_{n}^{2}}
$$


and so

$$
\left|\int_{\mathbb{T}} h_{2}(z) g(z) \mathrm{d} z\right| \leq 2 \pi|f|_{X_{N}}|g|_{X_{N}} \sum_{n=1}^{\infty} \frac{r_{n}}{s_{n}^{2}} .
$$

The estimate for the $h_{1}$ integral requires an application of Fubini's theorem: we have

$$
\begin{gathered}
\int_{\mathbb{T}} h_{1}(z) g(z) \mathrm{d} z=\int_{\mathbb{T}}\left(\frac{1}{2 \pi i} \int_{\gamma_{1}} \frac{f(w)}{(w-z)^{2}} \mathrm{~d} w\right) g(z) \mathrm{d} z \\
=\frac{1}{2 \pi i} \int_{\gamma_{1}} f(w)\left(\int_{\mathbb{T}} \frac{g(z)}{(w-z)^{2}} \mathrm{~d} z\right) \mathrm{d} w .
\end{gathered}
$$

However, we may apply Cauchy's theorem to the function $g(z) /(w-z)^{2}$ to obtain

$$
\int_{\mathbb{T}} \frac{g(z)}{(w-z)^{2}} \mathrm{~d} z=-\int_{\gamma_{2}} \frac{g(z)}{(w-z)^{2}} \mathrm{~d} z
$$

and so, by (2),

$$
\left|\int_{\mathbb{T}} g(z) /(w-z)^{2} \mathrm{~d} z\right| \leq|g|_{X_{N}} \sum_{n=1}^{N} 2 \pi \frac{r_{n}}{s_{n}^{2}} .
$$

Substituting this into equation (4) gives us

$$
\left|\int_{\mathbb{T}} h_{1}(z) g(z) \mathrm{d} z\right| \leq 2 \pi \frac{N+1}{N}|f|_{X_{N}}|g|_{X_{N}} \sum_{n=1}^{\infty} \frac{r_{n}}{s_{n}^{2}} .
$$

Combining (1), (3) and (5) gives

$$
\left|\int_{\gamma} f^{\prime}(z) g(z) \mathrm{d} z\right| \leq(4 \pi+\varepsilon)|f|_{X}|g|_{X} \sum_{n=1}^{\infty} \frac{r_{n}}{s_{n}^{2}}
$$

Letting $\varepsilon$ tend to 0 gives the result.

The next two results enable us to eliminate bounded point derivations by first working on compact subdiscs of $\Delta$.

Lemma 2.2. Let $X$ be a compact subset of $\bar{\Delta}$. Suppose that there is a sequence of real numbers $R_{n} \in(0,1)$ such that $R_{n} \rightarrow 1$ and, for each $n, R\left(X \cap \bar{\Delta}\left(0, R_{n}\right)\right)$ has no nonzero, bounded point derivations. Then $R(X)$ has no nonzero, bounded point derivations.

Proof. It is clear that all points of $X \cap \mathbb{T}$ are peak points, and so there are no nonzero point derivations at all at these points. For the remaining points, the fact that $R(X)$ has no nonzero, bounded point derivations follows from the local nature of this property, using (for example) Hallstrom's theorem ([12 Theorem 1]).

The next lemma may be proved quickly by elementary arguments. It is also, however, an immediate consequence of Hallstrom's theorem.

Lemma 2.3. Let $Y, Z$ be compact plane sets with $Y \subseteq Z$. If $R(Z)$ has no nonzero, bounded point derivations, then the same is true for $Y$.

Finally we quote a result proved by Wermer [17] (see also [12]).

Proposition 2.4. Let $D$ be a closed disc in $\mathbb{C}$ and let $\varepsilon>0$. Then there is a sequence of open discs $U_{k} \subseteq D$ such that $R\left(D \backslash \bigcup_{k=1}^{\infty} U_{k}\right)$ has no nonzero, bounded point derivations but such that the sum of the radii of the discs $U_{k}$ is less than $\varepsilon$. 
We are now ready to construct the Swiss cheese we want.

Proof of Theorem 1.1. Let $C>0$. Set $R_{n}=n /(n+1)$. By Proposition 2.4 we may choose open discs $U_{n, k} \subseteq \bar{\Delta}\left(0, R_{n}\right)$ such that $R\left(\bar{\Delta}\left(0, R_{n}\right) \backslash \bigcup_{k=1}^{\infty} U_{n, k}\right.$ has no nonzero, bounded point derivations and such that the sum (over $k$ ) of the radii of the discs $U_{n, k}$ is less than $C\left(1-R_{n}\right)^{2} /\left(2^{n+3} \pi\right)$.

Set

$$
X=\bar{\Delta} \backslash \bigcup_{n, k} U_{n, k} .
$$

Lemmas 2.2 and 2.3 show that $R(X)$ has no nonzero, bounded point derivations. For the rest, we may enumerate all the discs $U_{n, k}$ as $D_{1}, D_{2}, D_{3}, \ldots$ and then apply Lemma 2.1 to obtain the required estimate on the integral.

We conclude this section by discussing some additional properties of the uniform algebra $R(X)$ and the derivation $D: R(X) \rightarrow R(X)^{\prime}$ constructed using Theorem 1.1 (for some $C>0$ ). It is easily seen that this derivation is cyclic. This means that it satisfies $(D(f))(g)=-(D(g))(f)$ for all $f, g$ in $R(X)$ (or, equivalently, $(D(f))(1)=0$ for all $f \in R(X))$.

Notation. Let $A$ be a uniform algebra on a compact Hausdorff space $X$, and let $E$ be a closed subset of $X$. We denote the ideal of functions in $A$ vanishing identically on $E$ by $I(E)$.

In this setting it is standard that the restriction algebra $A \mid E$ is isomorphic to $A / I(E)$ and so is a Banach function algebra using the quotient norm.

Taking $A$ to be our example of $R(X)$ above, the derivation $D$ constructed shows that $A$ is not weakly amenable. We now show that the Banach function algebra $A \mid \mathbb{T}$ is not weakly amenable either. Since the derivation above is defined on elements of $R_{0}(X)$ using only their values on $\mathbb{T}$, we would expect to be able to use this to define a derivation on $A \mid \mathbb{T}$. However, some caution is needed: so far we only know, for $f, g$ in $R(X)$, that $|(D(f))(g)| \leq C|f|_{X}|g|_{X}$. For $f, g$ in $R_{0}(X)$, though, we have $|D(f)(g)| \leq 2 \pi\left|f^{\prime}\right|_{\mathbb{T}}|g|_{\mathbb{T}}$. Since, as we mentioned above, $D$ is cyclic, we also have $|D(f)(g)| \leq 2 \pi|f|_{\mathbb{T}}\left|g^{\prime}\right|_{\mathbb{T}}$. It now follows by continuity, for $f, g$ in $R(X)$, that if at least one of $f, g$ is in $I(\mathbb{T})$, then $D(f)(g)=0$. Thus, using some elementary functional analysis, we may define a nonzero, continuous derivation $\tilde{D}$ from the Banach function algebra $A \mid \mathbb{T}$ to $(A \mid \mathbb{T})^{\prime}$ by $(\tilde{D}(f \mid \mathbb{T}))(g \mid \mathbb{T})=(D(f))(g)$.

This shows that $A \mid \mathbb{T}$ is not weakly amenable. Since weak amenability passes to quotients (by, for example, [11] Proposition 2.1]), it follows that any other uniform algebra with a restriction isomorphic to $A \mid \mathbb{T}$ must also fail to be weakly amenable. This observation will be crucial in the next section.

\section{Peak Points Without Weak amenability}

The main result of this section will be the following theorem.

Theorem 3.1. There exists a uniform algebra $B$ whose maximal ideal space is metrizable (so $A$ is separable) and such that every point of the maximal ideal space of $B$ is a peak point (equivalently, every maximal ideal has a bounded approximate identity), but such that $B$ is not weakly amenable.

To construct this algebra, we will need a slightly modified version of the example constructed above, ensuring that there is a good supply of functions in the algebra 
vanishing identically on the circle. We will then use a version of Cole's systems of root extensions (see [5] and also [7]) to obtain the result.

We begin with some definitions and lemmas that we need. In particular, we will prove a result concerning the preservation of regularity properties by certain types of algebra extension, including Cole's root extensions. (See [7] for some other results of this type.)

Notation. Let $A$ be a uniform algebra on a compact space $X$. For $x \in X$, we denote by $M_{x}$ and $J_{x}$ the ideals of functions in $A$ vanishing at $x$, and in a neighbourhood of $x$, respectively.

We now recall the definition of a point of continuity (introduced in [9]).

Definition 3.2. Let $A$ be a uniform algebra on a compact Hausdorff space $X$, and let $x \in X$. We say that $x$ is a point of continuity for $A$ if there is no point $y$ of $X \backslash\{x\}$ satisfying $M_{x} \supseteq J_{y}$.

Equivalently, $x$ is a point of continuity for $A$ if and only if, for every compact set $E \subseteq X \backslash\{x\}$, there is a function $f$ in $A$ such that $f(x)=1$ and $f(E) \subseteq\{0\}$.

The algebra $A$ is regular on $X$ if every point of $X$ is a point of continuity for $A$. In this case, if $X$ is the maximal ideal space of $A$, then $A$ is regular.

See 8 for details of the connections between points of continuity and Jensen measures.

In the case where $E=\emptyset$, the next result follows from the work of Cole ([5]), since every representing measure must then be a Jensen measure. Recall also that, for metrizable spaces, strong boundary points and peak points coincide. In particular, this will be the case when we prove Theorem 3.1.

Lemma 3.3. Let $A$ be a uniform algebra on a compact space $X$, and let $x$ be a point of continuity for $A$. Suppose that $E$ is a compact subset of $X \backslash\{x\}$ such that $\left\{f^{2}: f \in I(E)\right\}$ is dense in $I(E)$. Then $x$ is a strong boundary point for A.

Proof. Note first, for convenience, that an easy application of the Mittag-Leffler inverse limit theorem (4, Corollary A.1.25]) shows that $I(E)$ has a dense subset consisting of functions with roots (in $A$, and hence in $I(E)$ ) of order $2^{k}$ for all $k \in \mathbb{N}$. Call this set of functions $S$.

Let $U$ be an open neighbourhood of $x$. We show that there is a function $f \in A$ with $|f|_{X \backslash U}<1 / 3,|f(x)|>2 / 3$ and $|f|_{X}<2$. The result then follows from Gonchar's criterion ([16, Corollary 7.20]).

Since $x$ is a point of continuity for $A$, we may choose a function $g \in A$ such that $g(x)=1, g(X \backslash U) \subseteq\{0\}$ and $g(E) \subseteq 0$. Set $M=\|g\|_{X}$.

Choose $k \in \mathbb{N}$ such that $M^{2^{-k}}<2$, and then choose $\delta \in(0,1)$ such that $\delta^{2^{-k}}<1 / 3,(M+\delta)^{2^{-k}}<2$ and $(1-\delta)^{2^{-k}}>2 / 3$. Now choose $h \in S$ with $|h-g|_{X}<\delta$ and choose $f \in A$ with $f^{2^{k}}=h$. It is clear that the function $f$ has the required properties.

The next lemma shows that in some sense the property of being a point of continuity is preserved by certain types of algebra extension. (Cole's systems of root extensions are of this type.) Note that we make no assumptions on the maximal ideal spaces of $A$ and $B$ here. 
Lemma 3.4. Let $A, B$ be uniform algebras on, respectively, compact spaces $X$, $Y$, and suppose that there are a continuous surjection $\pi: Y \longrightarrow X$ and $x_{0} \in X$ satisfying:

(a) for all $f \in A, f \circ \pi$ is in $B$;

(b) the points of the fibre $F=\pi^{-1}\left(x_{0}\right)$ are separated by a family of functions in $B$ each of which takes only finitely many different values on this fibre $F$ (so, in particular, $F$ is totally disconnected).

If $x_{0}$ is a point of continuity for $A$, then every point of the fibre $F$ is a point of continuity for $B$.

Proof. Let $x \in F$ and $y \in Y \backslash\{x\}$. We show that there exists $g$ in $B$ vanishing on some neighbourhood of $y$ but with $g(x) \neq 0$.

First, consider the case where $y$ is not in $F$. Then $\pi(y) \neq x_{0}$. Since $x_{0}$ is a point of continuity for $A$, we may choose an $f \in A$ that vanishes on an open neighbourhood $U$ of $\pi(y)$ and with $f\left(x_{0}\right) \neq 0$. Take $g=f \circ \pi$. Then $g$ vanishes on the open neighbourhood $\pi^{-1}(U)$ of $y$, and $g(x) \neq 0$, as required.

It is now easy to see that for every compact set $E \subseteq Y \backslash F$ there is a function $h$ in $B$ vanishing on $E$ and with $h(x) \neq 0$.

We now consider the remaining case, where $y \in F$. By the assumption (b) there is a function $b \in B$ taking only finitely many values on $F$ and with $b(x) \neq b(y)$. Applying a polynomial to $b$ if necessary, we may assume that $b$ takes only the values 0 and 1 on $F, b(y)=0$ and $b(x)=1$.

Choose an open neighbourhood $V$ of $F$ such that $b(V) \subseteq \Delta(0,1 / 3) \cup \Delta(1,1 / 3)$. By Runge's theorem we may choose a sequence of polynomials $p_{n}$ converging uniformly on $\Delta(0,1 / 3) \cup \Delta(1,1 / 3)$ to $\chi_{\Delta(1,1 / 3)}$. Choose $h \in B$ vanishing on $Y \backslash V$ and with $h(x) \neq 0$. Then the functions $h p_{n}(b)$ converge uniformly on $Y$ to a function $g$. We then have that $g$ is in $B, g(x)=h(x) \neq 0$, and $g$ vanishes identically on the neighbourhood $b^{-1}(\Delta(0,1 / 3))$ of $y$, as required.

We are now ready to prove Theorem 3.1. In the interests of conciseness we leave some details to the reader. For further details on systems of root extensions the reader may wish to consult [5], [16, Section 19] and, for a similar construction, 7 . Section 5].

Proof of Theorem 3.1. Using methods similar to those of 8] (based on Körner's version of McKissick's lemma, [14]) we may easily modify the Swiss cheeses constructed in the proof of Theorem 1.1 to ensure, in addition, that every point of $X \backslash \mathbb{T}$ is a point of continuity for $R(X)$.

For such a compact plane set $X$ we set $A=R(X)$, and form a Cole system of root extensions as follows. We first adjoin square roots to a countable dense set of functions in $I(\mathbb{T})$. This ensures that a copy of $\mathbb{T}$ persists in the maximal ideal space of the new uniform algebra. We next adjoin square roots to a countable dense subset of the ideal of functions in the new uniform algebra vanishing on this copy of $\mathbb{T}$. Repeating this process, we obtain a sequence of uniform algebras, and we take the direct limit of this sequence. This produces a uniform algebra $B$ on a compact metrizable space $Y$ and a continuous surjection $\pi$ from $Y$ onto $X$ with the following four properties.

(i) Conditions (a) and (b) of Lemma 3.4 hold for all $x_{0}$ in $X$.

(ii) Set $E=\pi^{-1}(\mathbb{T})$. Then $\pi \mid E$ is a homeomorphism from $E$ onto $\mathbb{T}$. 
(iii) There is a norm-decreasing linear map $T$ from $B$ onto $A$ (obtained by averaging over fibres) such that $T(f \circ \pi)=f$ for all $f \in A$.

(iv) With $E$ as above, $\left\{f^{2}: f \in I(E)\right\}$ is dense in $I(E)$.

We claim that the uniform algebra $B$ has the desired properties. First note that, for $g \in B$, we have $g \mid E=(T(g) \mid \mathbb{T}) \circ \pi$. It follows easily that the restriction algebras $A \mid \mathbb{T}$ and $B \mid E$ are isomorphic. By the comments at the end of Section 2, this shows that $B$ is not weakly amenable.

We wish to show that every point of $Y$ is a peak point for $B$. For points in $E$ this is elementary, since we may use $f \circ \pi$ for appropriate peaking functions in $A$. The remaining points lie in the fibres over points of continuity for $A$, and so the result follows from Lemmas 3.3 and 3.4.

\section{Open questions}

We conclude with some open questions.

(1) Let $X$ be a compact plane set. Suppose that $R(X)$ is weakly amenable. Must $R(X)=C(X)$ ?

(2) Are there any nontrivial, weakly amenable uniform algebras?

(3) Let $X$ be a compact plane set. Suppose that $R(X)$ has no nonzero, bounded point derivations. Can there ever be a measure $\mu$ on a compact subset $E$ of $X$ such that the map $f \mapsto f^{\prime} \mid E$ gives a nonzero, bounded derivation from $R_{0}(X)$ into $L^{1}(E, \mu)$ ?

\section{REFERENCES}

[1] W. G. Bade, P. C. Curtis, Jr., and H. G. Dales, Amenability and weak amenability for Beurling and Lipschitz algebras, Proc. London Math. Soc. (3) 55 (1987), no. 2, 359-377. MR 88f:46098

[2] F. F. Bonsall and J. Duncan, Complete Normed Algebras, Springer-Verlag, New York, 1973. MR 54:11013

[3] A. Browder, Introduction to Function Algebras, W. A. Benjamin, Inc., New York, 1969. MR 39:7431

[4] H. G. Dales, Banach algebras and automatic continuity, London Mathematical Society Monographs, New Series, 24, Oxford Science Publications, The Clarendon Press, Oxford University Press, New York, 2000. MR 2002e:46001

[5] B. J. Cole, One point parts and the peak point conjecture, Ph.D. Thesis, Yale University, 1968.

[6] J. F. Feinstein, Weak $(F)$-amenability of $R(X)$, Proceedings of the Centre for Mathematical Analysis, Australian National University, Canberra, 21 (1989), 97-125. MR 91h:46092

[7] J. F. Feinstein, A nontrivial, strongly regular uniform algebra, J. London Math. Soc., 45 (1992), 288-300. MR 93i:46086

[8] J. F. Feinstein, Trivial Jensen measures without regularity, Studia Mathematica, 148 (2001) 67-74. MR 2002k:46127

[9] J. F. Feinstein and D. W. B. Somerset, Non-regularity for Banach function algebras, Studia Mathematica, 141 (2000), 53-68. MR 2001g:46115

[10] T. W. Gamelin, Uniform Algebras, Prentice-Hall, Inc., Englewood Cliffs, NJ, 1969. MR 53:14137

[11] N. Grøenbæck, A characterization of weakly amenable Banach algebras, Studia Math., 94 (1989), 149-162. MR 92a:46055

[12] A. P. Hallstrom, On bounded point derivations and analytic capacity, Journal of Functional Analysis, 4 (1969), 153-165. MR 39:4680

[13] B. E. Johnson, Cohomology in Banach algebras, Memoirs of the American Mathematical Society, No. 127, Providence, RI, 1972. MR 51:11130

[14] T. W. Körner, A cheaper Swiss cheese, Studia Math., 83 (1986), 33-36. MR 87f:46090 
[15] S. E. Morris, Bounded derivations from uniform algebras, Ph.D. thesis, University of Cambridge, 1993.

[16] E. L. Stout, The Theory of Uniform Algebras, Bogden and Quigley, Tarrytown-on-Hudson, New York, 1971. MR 54:11066

[17] J. Wermer, Bounded point derivations on certain Banach algebras, J. Funct. Anal., 1 (1967), 28-36. MR 35:5948

School of Mathematical Sciences, University of Nottingham, Nottingham NG7 2RD, ENGLAND

E-mail address: Joel.Feinstein@nottingham.ac.uk 